\title{
Addendum
}

\section{Quantum Tunneling at Low Temperatures: Results for Weak Damping}

\author{
E. Freidkin and P.S. Riseborough \\ Department of Physics, Polytechnic Institute of New York, \\ Brooklyn, New York, USA \\ P. Hänggi \\ Lehrstuhl für Theoretische Physik, Universität Augsburg, \\ Federal Republic of Germany
}

Received March 18, 1986

Z. Phys. B - Condensed Matter 64, 237-246 (1986)

The approximate evaluations of the intergrals in (4.8) and (4.12) can be improved. These integrals can be performed exactly, with the results that in Eq. (4.13) the factor 1.16 should be replaced by 0.472 and 4.07 should be replaced by 0.056 . In Eq. (4.14) the factor 1.60 should be precisely 0 . The contribution of the continuum eigenvalues can be evaluated directly with Eq. (4.12). As a result of this, Eq. (4.21) should be replaced by $15 / 2[1+3.011 \propto]$. On combining these contributions, one finds a factor of 2.860 instead of 5.47 in (4.23). Finally, a factor of $\exp \left[i k \omega_{0} \tau / 2\right]$ should be included in (A.5) of Appendix 1.
E. Freidkin, P.S. Riseborough Department of Physics Polytechnic Institute of New York 333 Jay Street

Brooklyn, NY 11201

USA

P. Hänggi

Lehrstuhl für Theoretische Physik

Universität Augsburg

Memmingerstrasse 6

D-8900 Augsburg

Federal Republic of Germany 